\title{
Moisture induced straining of the cellulosic microfibril
}

\author{
Lennart Salmén (iD) - Jasna S. Stevanic • Claes Holmqvist • Shun Yu
}

Received: 6 July 2020/ Accepted: 22 January 2021 / Published online: 2 March 2021

(C) The Author(s) 2021

\begin{abstract}
Moisture absorption in the cell wall structure of wood is well known to induce considerable swelling of the wood exerting high expansion forces. This swelling is mainly induced by the sorptive action of the hydroxyl groups of the carbohydrate wood polymers; cellulose and hemicelluloses. On the ultrastructural level, there are, however, still questions with regard to the detailed deformations induced by this moisture absorption. Here, FTIR spectroscopy and synchrotron-radiation-based X-ray diffraction were used on paper samples to study the deformation of the cellulose crystals as a consequence of moisture absorption and desorption. Both techniques revealed that the moisture absorption resulted in a transverse
\end{abstract}

contraction of the cellulose crystals accompanied by a somewhat smaller elongation in the cellulose chain direction. The deformations were found to be a direct response to the increased moisture content and were also found to be reversible during moisture desorption. It is hypothesised that these deformations are a consequence of the swelling forces created by the combined longitudinal and lateral expansions of the non-crystalline cellulose molecules and the glucomannan hemicellulose aligned along the cellulose crystals. These forces will impose a lateral contraction of the cellulose crystals, as well as a longitudinal extension of it.
Supplementary Information The online version contains supplementary material available at https://doi.org/10.1007/ s10570-021-03712-1.

L. Salmén $(\bowtie)$ · J. S. Stevanic $(\bowtie)$.

C. Holmqvist $\cdot \mathrm{S}$. Yu

RISE Bioeconomy and Health, Box 5604, 11486 Stockholm, Sweden

e-mail: 1salmen@kth.se

J. S. Stevanic

e-mail: jasna.stevanic@ri.se 


\section{Graphic abstract}

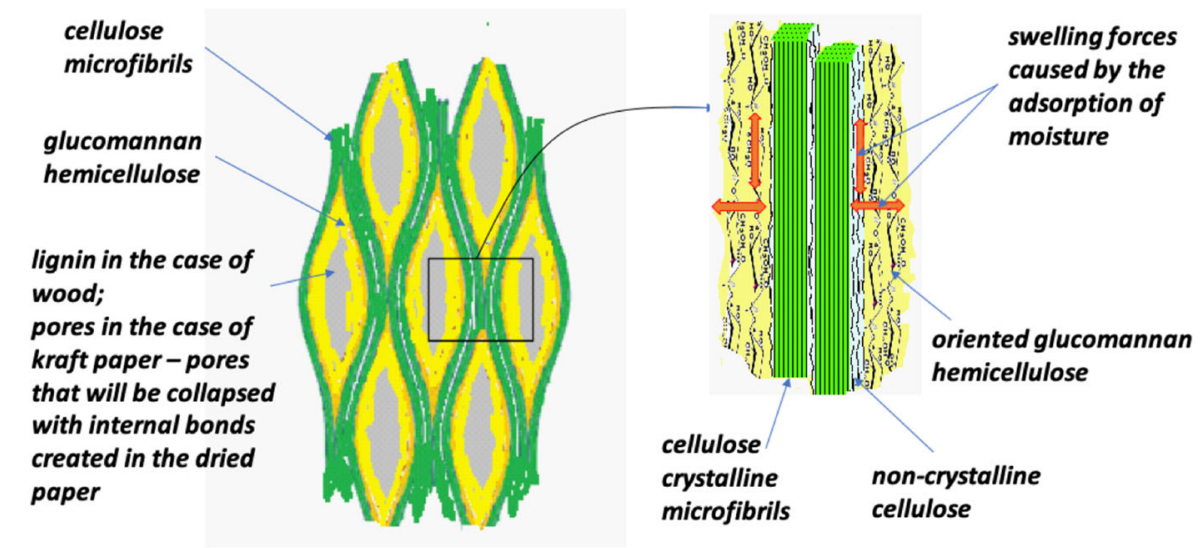

Keywords Cellulose · FTIR · Moisture changes · Paper $\cdot$ Relative humidity $\cdot \mathrm{X}$-ray diffraction

\section{Introduction}

Cellulose, as a biopolymer, is naturally affected by moisture and the relative content of it, the relative humidity ( $\mathrm{RH})$, in the environment. This is highly related to the molecular structure of the cellulose as containing a high amount of specific sorption sites, the hydroxyl groups $(-\mathrm{OH})$, which attract the water molecules. For decades, a great deal of research has been devoted to the question of how and where the water molecules attach to the cellulose molecules (Lindh et al. 2017). What role the biological structural arrangement in the cell wall has, as well as how moisture influences the properties of this material are questions addressed. The problem is related to the very complex structure of the cellulose microfibrils (also referred to as fibrils in literature), formed by biological processes, the fine structure of which is not fully established despite 100 years of studies (Salmén 2015). As a structure, the cellulose is arranged in the form of extended microfibrils, generally considered to have a diameter of around 3.0 to $3.5 \mathrm{~nm}$ (Fernandes et al. 2011). The cellulose molecules extend in the direction of the microfibril possessing both crystalline areas as well as less ordered, non-crystalline areas. To a large extent, the less ordered regions have been assigned to the surface of the microfibril (Wickholm et al. 1998), but areas interspersed between the cellulose crystalline domains along the microfibrils have been indicated (Nishiyama 2009). The possible twisting of the microfibrils (Fernandes et al. 2011) could also contribute to non-regular structures in the longitudinal direction. Considering this complex structure, it is understandable that the question of interaction with moisture poses a challenge. For the crystalline part of the cellulose, it is accepted, however, that moisture is not able to enter in-between cellulose molecules and disrupt the crystallites (Nishiyama et al. 2002). Studies of the deformation of cellulose microfibrils under different moisture conditions using dynamic FTIR spectroscopy indicate that the deformation follows a relation of an arrangement of parallel non-crystalline and crystalline structures (Salmén and Bergström 2009), i.e. a structure where the less-ordered regions are dominantly positioned at the surface of the microfibrils. This is corroborated by the fact, that for low microfibril angles $\left(\mathrm{MFA}<10^{\circ}\right.$ ), more than $95 \%$ of the strain of small wood elements exposed to a tensile stress is given by the strain of the crystalline cellulose (Nakai et al. 2006). Thus, it may be envisaged that noncrystalline structures arranged in series with crystalline sections must have a rather limited extension, possibly because of adjacent crystalline microfibrils, which prevent them from being highly strained. Such a structural interlocking may presumably be due to the aggregated structure of the cellulose microfibrils, on average making up a cluster of a diameter around 16 nm (Bardage et al. 2004; Fahlén and Salmén 2005). In this structure, moisture interacts both with the outer cellulose aggregate surfaces as well as with the interior 
surfaces of the aggregate, though to different extents (Lindh and Salmén 2017; Lindh et al. 2017; Salmén and Stevanic 2018). Thus, less mobile water will be found in-between the cellulose fibrils in the aggregate while more mobile water is located at the outer aggregate surfaces.

The effect of the added water to the properties of the cellulose is well known on a macroscopic level, while its interactions at the supramolecular level are not so frequently discussed and still poses questions. Macroscopically the uptake of moisture leads to a weakening of the structure of the material, lower stiffness, and an expansion occurring mostly transverse to the cellulose microfibrils (Megraw 1985; Salmén 2004). Indeed, the moisture expansion along microfibrils is less than a tenth of that in the transverse direction. On the cellulose crystalline level, it has been observed that drying of wood samples leads to a longitudinal shrinkage of the cellulose crystal (Abe and Yamamoto 2005). Synchrotron radiation (SR)-based X-ray diffraction studies of spruce wood have additionally revealed that there is a correlation between the expansion of the cellulose crystal chains in the longitudinal direction and the increased equilibrium moisture content below a moisture content of $15 \%$ (Zabler et al. 2010). Accompanying this is a shrinkage of the transverse dimension of the cellulose crystal, a dimensional change which thus is opposite to the one observed on a macroscopic, fibre scale although the magnitude is much smaller (Zabler et al. 2010). Thus, there are still questions regarding the causes of these movements as well as the kinetics of the changes. On the macroscopic level, it has been shown that for paper the dimensional changes are directly coupled to the amount of moisture sorbed and occur instantaneously with the moisture uptake (Salmén and Olsson 2016). In order to in-situ follow such changes in the deformation of the cellulose structure, two useful techniques may be identified: FTIR spectroscopy and SR-based X-ray diffraction. Both techniques offer the possibility of detecting changes in the molecular or crystalline structure with the time-resolution required for absorption-desorption studies, i.e. responses in the region of seconds. Here, both techniques have been applied to paper samples to identify the response to moisture changes at the cellulose molecular and structural level.

\section{Materials and methods}

Materials

Paper sheets for FTIR spectroscopy studies were made from an unbleached softwood kraft pulp with a kappa number of 58 and produced in a Finnish sheet former at $20 \mathrm{~g} / \mathrm{m}^{2}$ (ISO 5269-1). In order to facilitate IR transmission, samples with a thickness of about $30 \mu \mathrm{m}$ were employed. These were cut into the dimensions of $18 \times 25 \mathrm{~mm}$ (width-length).

Paper sheets for SR-based X-ray diffraction studies were made from a chlorite delignified latewood (LW) fibre enriched unbleached softwood kraft pulp (39\% LW fibres as compared to an original unfractionated pulp of $28 \% \mathrm{LW}$ fibres). The pulp fibres were beaten in a PFI laboratory beater to 5000 revolutions, yielding a Schopper-Riegler (SR, ISO 5267-1) value of 24. Oriented sheets with a target dry basis weight of $45 \mathrm{~g} / \mathrm{m}^{2}$ were made with a Dynamic Sheet Former laboratory apparatus from Fibertech (a.k.a. "French sheet former"). The purpose was here to produce a paper sheet with cellulose fibrils with the preferred orientation; high fibre orientation and low microfibrillar angle (MFA, fibrils aligned in the fibre direction), with a high cellulose content to provide for suitable SR-based X-ray diffraction studies. Samples with a thickness of $50 \mu \mathrm{m}$ were cut with the dimensions of $15 \times 35 \mathrm{~mm}$ (width-length in the preferred orientation direction).

Methods

FTIR (Fourier Transform Infra-Red) spectroscopy was performed using a Varian 680-IR.

FTIR spectrometer (Varian Inc., Santa Clara, CA, USA), equipped with a DTGS detector and run in transmission mode. The IR radiation was polarized by a KRS5 wire grid polarizer at $0^{\circ}$ in relation to the stretching direction of the samples. Samples were mounted between two parallel jaws, with an initial separation of $15 \mathrm{~mm}$, in a specially constructed Polymer Modulator (PM100) (MAT-Manning Applied Technology Inc., Troy, ID, USA).

The stress in the samples was controlled using a PicoScope oscilloscope and PicoScope software (Pico Technology Ltd., St Neots Cambridgeshire, UK). A Temperature Control System (TC100) (MATManning Applied Technology Inc., Troy, ID, USA) 
was used for controlling the temperature $\left(30^{\circ} \mathrm{C}\right)$ inside the PM100.

A Modular Humidity Generator (MHG 32) (Project Messtechnik, Ulm, Germany) was used for generating the humid atmosphere, which was connected to the TC100 with a mounted sample. Humidity was controlled using MHG Control software (Project Messtechnik, Ulm, Germany).

Tests were performed during an absorption-desorption cycle from $30 \% \mathrm{RH}$ to $80 \% \mathrm{RH}$ and back again to $30 \% \mathrm{RH}$ with a cycle time of 30 minutes for adsorption and desorption, respectively. Prior to the test cycle the sample had been conditioned at $30 \% \mathrm{RH}$ for 2 hours at a low load of $1.5 \mathrm{~N}$ to avoid buckling of the sample sheet. All spectra during the sorption cycle were collected as unloaded (i.e. at low load of $1.5 \mathrm{~N}$ to avoid the buckling of the sample sheet). Background and sample spectra were scanned using a spectral resolution of $1 \mathrm{~cm}^{-1}$ and a spectral range of $4000-700 \mathrm{~cm}^{-1} ; 32$ scans were collected. Spectra were baseline corrected using a Varian Resolution Pro software. Spectra were normalized at $1160 \mathrm{~cm}^{-1}$ (cellulose glycosidic bond $(\mathrm{C}-\mathrm{O}-\mathrm{C})$ anti-symmetric stretching vibration).

All experimental cases were run in duplicate.

SR-based X-ray diffraction experiments were performed at the beamline P03 "MiNaXS" at Petra III storage ring at DESY, Hamburg, Germany. The experimental setup consisted of a tensile testing load stage (TS600) from Anton Paar GmbH, Austria, mounted in a casing for climate control, with Kapton windows for beam transmission. The sample strips were mounted in the tensile stage with an original clamp length of $24 \mathrm{~mm}$, fastened with only one clamp and run as completely unloaded (load $0 \mathrm{~N}$ ). Temperature and humidity controls were arranged by connecting the casing to a commercial programmable humidity control unit (MHG 32, see the above description for FTIR spectroscopy, only that prior to the test cycle the sample had been conditioned at $30 \%$ RH for 1 hour and at no-load, $0 \mathrm{~N}$ ).

The X-ray wavelength used was $0.1068 \mathrm{~nm}$. The WAXS (Wide Angle X-ray Scattering) detector was a tailor-made "L" shaped Lambda $2 \mathrm{M}$ detector developed by DESY, see Fig. 1. The system was calibrated in WAXS measuring mode using a reference sample of cerium dioxide $\left(\mathrm{CeO}_{2}\right)$. Background scans were collected at the constant humidities of $30 \%$ and $80 \%$ (a)

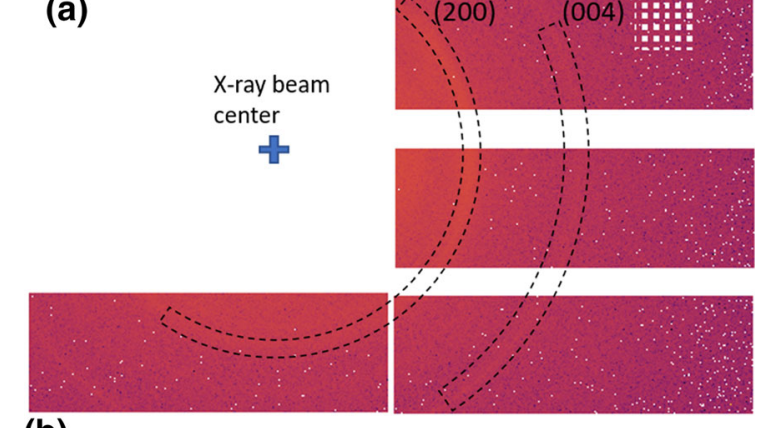

(b)

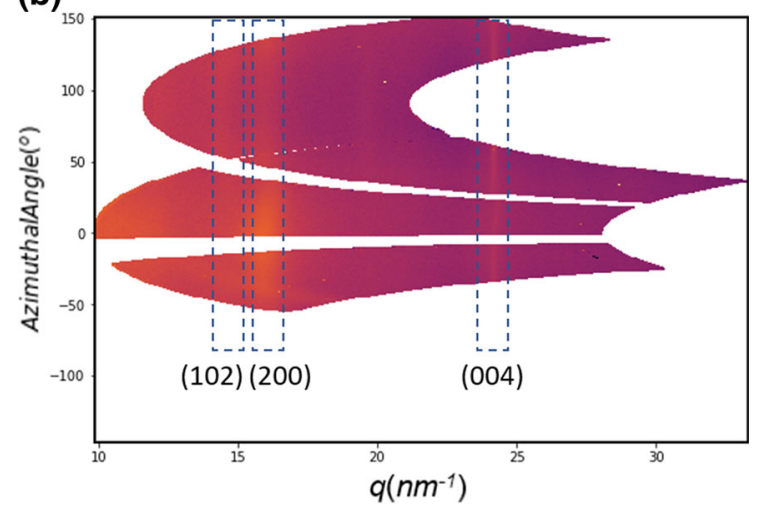

Fig. 1 a Lambda detector image; $\mathbf{b}$ azimuthal integrated image converted from a, reflection is marked inside the dashed box

RH using the empty TS600 encapsulated into the humidity chamber.

A large beam-size of approximately $100 \times 100$ $\mu \mathrm{m}^{2}$ was chosen for better statistics as the crosssection of late-wood fibres is around $30-40 \mu \mathrm{m}$. Collected images were scrutinized for X-ray beamdamage. The exposure time at each measurement position was then set to $1 \mathrm{~s}$ with the X-ray beam scanning over the sample to distribute the X-ray dose during the experiment. This was achieved by the movement of an $\mathrm{X}, \mathrm{Y}, \mathrm{Z}$ position table on which the sample stage was mounted.

Tests were performed during three humidity halfcycles (a first absorption half-cycle: $30 \% \rightarrow 80 \% \mathrm{RH}$, a desorption half-cycle: $80 \% \rightarrow 30 \% \mathrm{RH}$ and a second absorption half-cycle: $30 \% \rightarrow 80 \% \mathrm{RH}$ ); 10 scans were collected for each half-cycle, i.e. during $30 \mathrm{~min}$ at times arranged equally with respect to log time. In each scan, scattering data from 6 different positions across the sample width were acquired, after which the acquisition was moved to a new length-wise position for the next scan. The spacing between the positions was such that double exposure did not take place. 
The WAXS signals were analysed as 1D scattering profiles, i.e. diffractograms. The crystalline structure is shown as series scattering signals peaks (Fig. 2), where the signal maxima peak position, i.e. scattering vector $(q)$, is directly related to the $d$-spacing, the distance between adjacent lattice planes, Eq. (1):

$q=\frac{2 \pi}{d}$

The signal position $(q)$ is related to the scattering angle $(2 \theta)$ by:

$q=\frac{4 \pi}{\lambda} \sin (\theta)$

where $\lambda$ is the wavelength of the applied radiation (here $0.1068 \mathrm{~nm}$ ). Upon deformation, $d$ can be stretched or compressed, showing as a signal shift in the scattering patterns. By using the Eq. (3), the strain of the crystalline phase $\left(\epsilon_{d}\right)$ could be estimated as:

$\epsilon_{d}=\left(\frac{d}{d_{0}}-1\right) \bullet 100 \%$

where $d$ is the distance between the lattice planes determined from the scattering during deformation, $d_{0}$ is the one determined from the sample in the reference state.

Deformations of the crystals in different directions may be followed by monitoring of the different crystallographic lattice planes. Figure 2 illustrates how the cellulose unit cell (i.e. crystallite) relates to the corresponding scattering signals. A straining in the longitudinal direction of the cellulose molecule will result in an increase of the distances between the (004) planes and a shift in its scattering signal towards smaller angles. Also, the (102) planes will deform in a similar manner while the (200) planes will contract, shifting the associated scattering signal towards higher scattering angles due to the Poisson effect.

\section{Results and discussion}

FTIR spectroscopy measurements

The occurrence of changes in the FTIR spectra for a paper sample due to an increase in moisture content from 30 to $80 \%$ RH was clearly evident from the example of the difference spectrum shown in Fig. 3 (the original spectra shows the typical absorption peaks for wood pulps). The 30 to $80 \% \mathrm{RH}$ change corresponds to a change in moisture content from 4-5\% (depending on the cycle) to about 10 to $11 \%$. This moisture is only present as bound water in the material and will thus result in a corresponding volume expansion of the material (Salmén and Olsson 2016). A split signal (a signal with a peak both above and below 0 absorbance) was seen in the difference spectrum obtained by subtracting the spectrum taken at $80 \% \mathrm{RH}$ with that taken at the reference $30 \% \mathrm{RH}$. The split signal was due to the fact that the position of the $1162 \mathrm{~cm}^{-1}$ absorbance maximum was shifted to lower wavenumbers at the higher $\mathrm{RH}$, indicating that the bond length had been enlarged. The reference spectrum was taken at 30\% RH after a conditioning time of 2 hours. This clearly indicated that the absorbed moisture yielded stresses on the cellulose
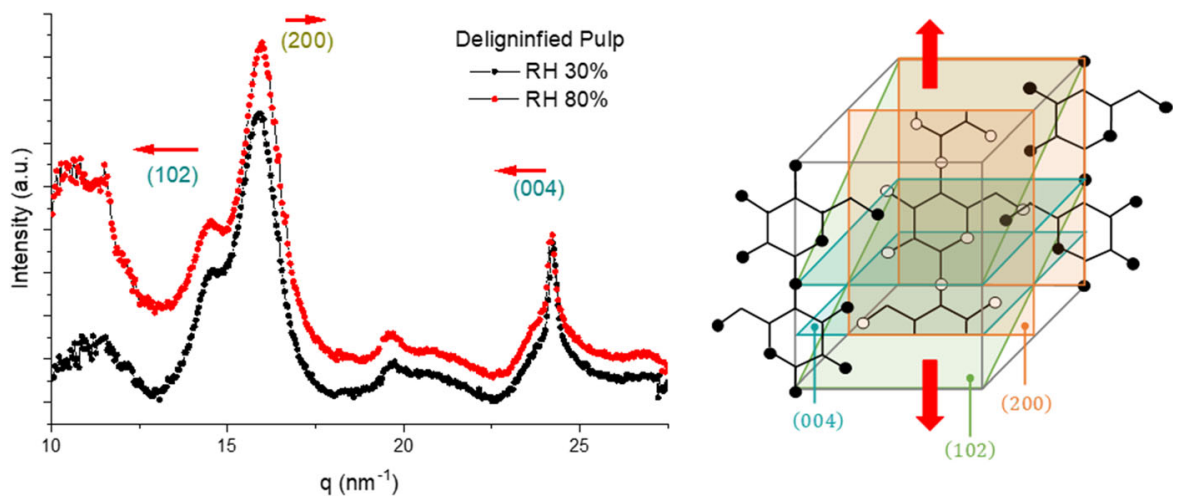

Fig. 2 Diffractogram (left) and crystallographic lattice plains of cellulose allomorph $1 \beta$ (i.e. delignified pulp) (right). The arrows indicate changes that would occur due to a straining of the cellulose crystal, i.e. the cellulose unit cell (or crystallite) in its longitudinal direction 
Fig. 3 Example of a difference spectrum (80-30\% RH) illustrating the effect of the relative humidity change for a paper sample under no-load (1.5 N applied only to straighten the paper) (lower diagram). The small shift of the peak position of the $1162 \mathrm{~cm}^{-1}$ signal of the original spectra is caused by the elongation of the $\mathrm{C}-\mathrm{O}-\mathrm{C}$ bond (upper diagram)
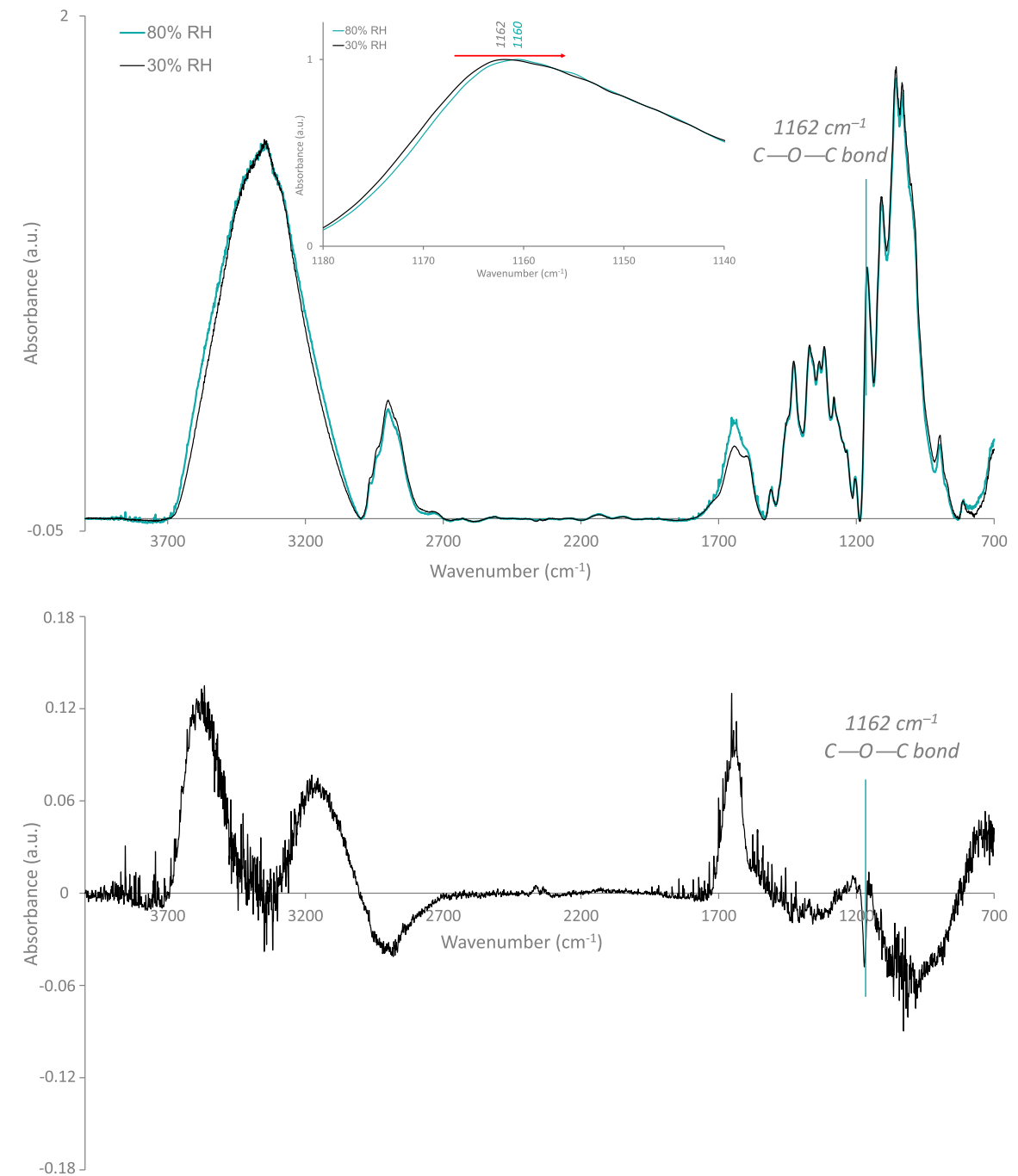

molecules resulting in the whole cellulose crystal being stretched. The additional changes seen in the spectrum were due to the absorbed moisture, two signals in the $\mathrm{OH}$-region of loosely and more strongly absorbed moisture at $3600 \mathrm{~cm}^{-1}$ and $3200 \mathrm{~cm}^{-1}$ respectively (Olsson and Salmén 2004), and the signal from the absorbed water itself at $1640 \mathrm{~cm}^{-1}$. The negative signals of the $\mathrm{CH}$-vibration at 3000 to $2700 \mathrm{~cm}^{-1}$ and the fingerprint region 1100 to $900 \mathrm{~cm}^{-1}$ are presumably related to the fact that the expansion of the paper sample leads to that less material is present within the IR beam and thus a lower absorbance.

In Fig. 4, the changes of the length of the cellulose glycosidic $\mathrm{C}-\mathrm{O}-\mathrm{C}$ bond as a result of the stepwise $\mathrm{RH}$ changes of $10 \%$ in a cycle from $30-80 \%$ and back to $30 \% \mathrm{RH}$ is illustrated. Here, the change was monitored as the absolute signal height (top to bottom) of the split signal. It should be noted that the signal height is a trigonometric function of the signal shift, related to the length change of the glycosidic $\mathrm{C}-\mathrm{O}-\mathrm{C}$ bond, and is hence not linearly related to the bond length. It nonetheless clearly shows that the cellulose molecules were strained in their longitudinal direction by the increasing $\mathrm{RH}$, the more so the higher the $\mathrm{RH}$, and that upon desorption the length retracted back towards the initial length. The discrepancy at $30 \%$ RH between the starting value and the one reached at the end of the desorption cycle was most probably related to the effect of moisture hysteresis, i.e. that the paper 
Fig. 4 Changes in height of the split absorption signal of the cellulose glycosidic $(\mathrm{C}-$ $\mathrm{O}-\mathrm{C})$ bond at $1162 \mathrm{~cm}^{-1}$, calculated from difference spectra between the actual spectrum and that at $30 \%$ $\mathrm{RH}$, as a function of $\mathrm{RH}$ during absorption and desorption

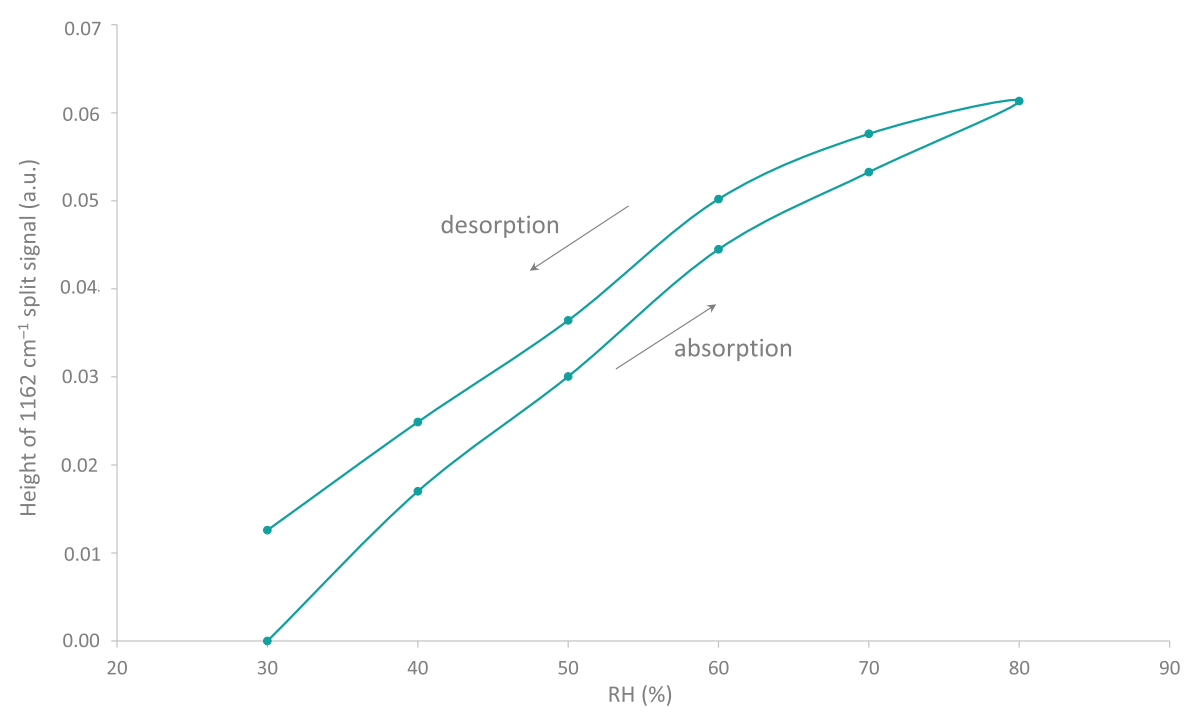

contains more moisture absorbed when coming from the higher RH than that reached when conditioned from lower RH values (Urquhart 1929).

\section{SR-based X-ray diffraction measurements}

From the synchrotron X-ray diffraction measurements, the lattice deformations as shown in Fig. 5 clearly indicate that moisture changes resulted in deformations of the cellulose crystals. The changes occurred quickly and were directly related to the change in relative humidity within the measuring chamber. The scattering from the (004) plane, indicating the length changes along the cellulose crystalline chains, clearly showed that with increasing RH, i.e. with increasing moisture content in the paper, the cellulose crystals were strained in the longitudinal direction. When the $\mathrm{RH}$ again reverted to $30 \% \mathrm{RH}$, the deformation retracted back at the same speed as the decrease of the RH. Simultaneous changes occurred for the (200) deformation, the transverse dimension of the crystals, which was clearly compressed and compressed to a larger extent than the longitudinal straining. The ratio of the magnitude of the strain along (004) and (200) was about 0.3 which is close to values reported on the ratio of these cellulose crystal deformations for wood samples under moisture desorption and reabsorption conditions (Zahler et al. (2010)). The evolution of the strain along (102), which is very similar to the (004) strain, clearly supports a deformation of the cellulose crystal in the longitudinal

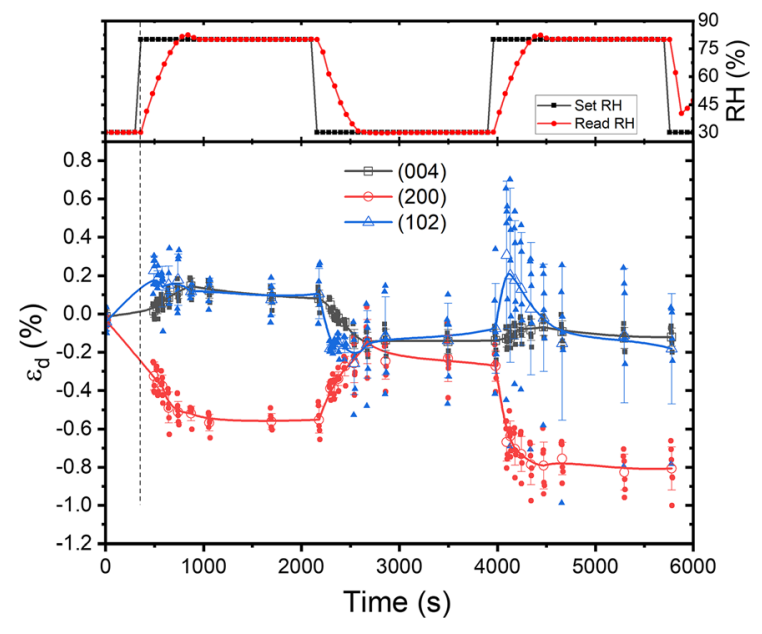

Fig. 5 Lattice strains of cellulose as a function of time during cyclic humidity changes between $30 \%$ and $80 \%$ RH under noload conditions $(0 \mathrm{~N})$. The $\mathrm{RH}$ was monitored within the measuring chamber (red curve in the top panel) with the dashed line marking the onset of $\mathrm{RH}$ change. Approximately 10 minutes was required for the RH to reach the target value. The (004) strain represents the longitudinal crystal direction while the (200) strain represents the transverse direction. The (102) also represents a longitudinal deformation. The solid lines in the bottom panel are the mean values of those at 6 different positions at each sampling time, representing repeated measurement

direction. There also seemed to be some drift in the measurements, presumably due to some issue with the data sampling and recording as the drift is similar for all signals. The changes seen in the longitudinal and 
transverse directions of the cellulose crystals were thus similar to the SR-based X-ray diffraction observations made by Zahler et al. (2010) upon drying of wood samples.

\section{Origin of deformations}

From both the FTIR spectroscopy and SR-based X-ray diffraction measurements, it seems clear that increased moisture content induced a longitudinal deformation of the cellulose crystal, which was accompanied by a lateral contraction. As it is fully established that cellulose does not absorb moisture within its crystalline structure (Nishiyama et al. 2002), the deformations have to be related to swelling forces originating from other components in the cell wall. Both hemicelluloses and the non-crystalline parts of the cellulose do absorb moisture and consequently also swell, i.e. expand in relation to the volume of the absorbed water molecules. It could be argued that the non-crystalline cellulose components that are located at the surface of the cellulose crystals, when taking up moisture and swelling, could impose the observed longitudinal strain on the crystalline parts. The fact that the glucomannan hemicellulose is highly associated to the cellulose microfibrils and oriented parallel to the cellulose molecules (Stevanic and Salmén 2009) would impose additional swelling forces on the cellulose in its longitudinal direction. The lateral contraction of the cellulose crystals could then be a Poisson effect. Although earlier estimates indicated a very low Poisson's ratio of 0.1 for cellulose (Mark 1967), more recent studies give values of 0.3 to 0.46 based on measurements (Roberts et al. 1994; Nakamura et al. 2004) and force field simulations (Wu et al. 2014). However, considering deformations caused by a longitudinal straining would mean, in our case, a Poisson's ratio of $\varepsilon_{\text {transversal }} / \varepsilon_{\text {axial }}$ of 3.33 ; an impossible value.

On the macroscopic level the swelling of the noncrystalline material is manifested in the much higher expansion of wood in its radial and tangential directions than that in its longitudinal direction (Megraw 1985). This is not surprising as it is well established that the swelling of an anisotropic material occurs mainly in the direction of least stiffness, i.e. perpendicular to the longitudinal direction of the polymers (de Ruvo et al. 1976). Thus, one could envisage that such lateral forces could create a compression of the cellulose crystal and, due to the Poisson effect, impose the resulting longitudinal crystal extension. The Poisson's ratio would then, in our case, be $\varepsilon_{\text {axial }} /$ $\varepsilon_{\text {transversal }}=0.3$. However, when considering deformations induced by lateral compression or straining, the Poisson's ratio is only, based on force field simulations, in the range of 0.01 to 0.04 (Wu et al. 2014) (no experimental data has yet been published). This means that neither a longitudinal straining nor a lateral compression may alone be responsible for the observed deformations. As the swelling of the noncrystalline carbohydrates, the surface non-crystalline cellulose and the glucomannan, results in expansions both in their lateral direction as well as in their longitudinal direction, it is suggested that it is the combination of these swelling forces that result in the observed deformation of the cellulose crystals.

The fact that the measurements here performed on paper, i.e. detecting the deformations of the cellulose crystals in a structure where much of the lignin has been removed, were very similar to the results obtained on wood (Zabler et al. 2010) indicates that the structural forces due to the swelling should be independent of the lignin within the cell wall. In the cell wall structure of the kraft pulp fibre the removed lignin will leave empty pores behind, see Fig. 6. This is a consequence of the cellulose microfibrils/aggregates not being completely straight, but having an undulating arrangement with lenticular spaces interspersed with the matrix material (hemicelluloses and lignin) (Boyd 1982; Bardage et al. 2004). In the dried paper the empty lenticular spaces will be collapsed and to some extent bonded to each other, the structure is "hornified". Thus, in both cases, that of wood and that of pulp fibres, the swelling of the non-crystalline carbohydrates will impose lateral forces on the cellulose crystals. As illustrated in Fig. 6, the swelling forces created by the moisture uptake and expansion of these carbohydrates will cause both a lateral compression as well as an axial elongation of the cellulose crystals. Further studies should be performed in order to verify these hypotheses.

The high stiffness of the cellulose crystal is well documented generally considered to be in the range of 112 to $134 \mathrm{GPa}$ in the axial direction, and in the range of 18 to $25 \mathrm{GPa}$ in the transverse direction (Salmén 2004; Pakzad et al. 2012; Wu et al. 2014). However, one should also remember that in the transverse direction the cellulose crystal poses anisotropy, 
Fig. 6 Schematic structure of the undulating cellulose microfibrils with oriented glucomannan attached to the non-crystalline cellulose surface molecules. The adsorption of moisture in these structures will lead to both a lateral and a longitudinal expansion creating lateral compression forces as well as axial extension forces on the cellulose crystals

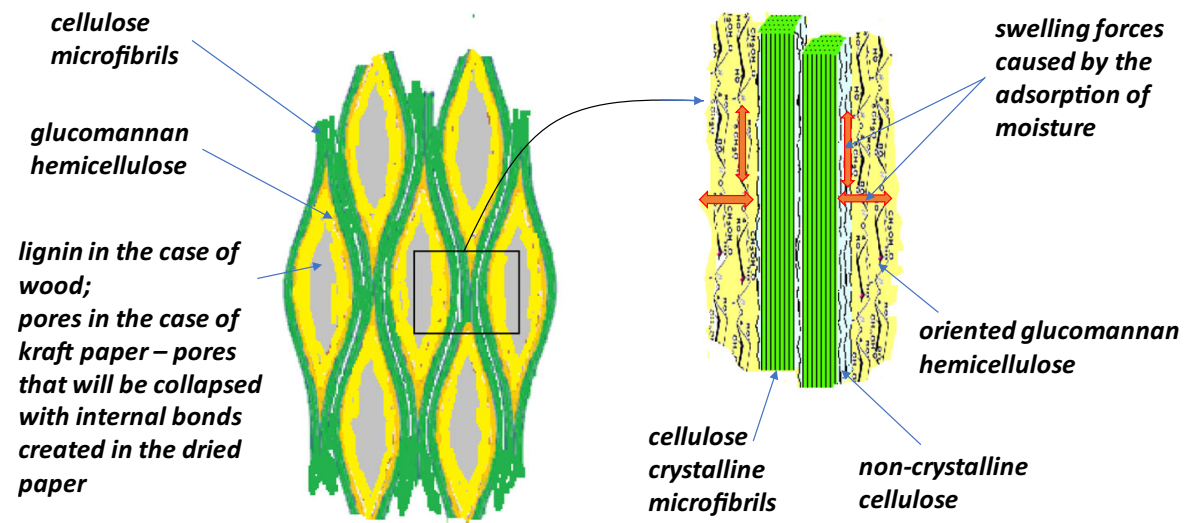

$-50$

0

Azimuthal Angle $\left({ }^{\circ}\right)$

50

100

150

$\Gamma$
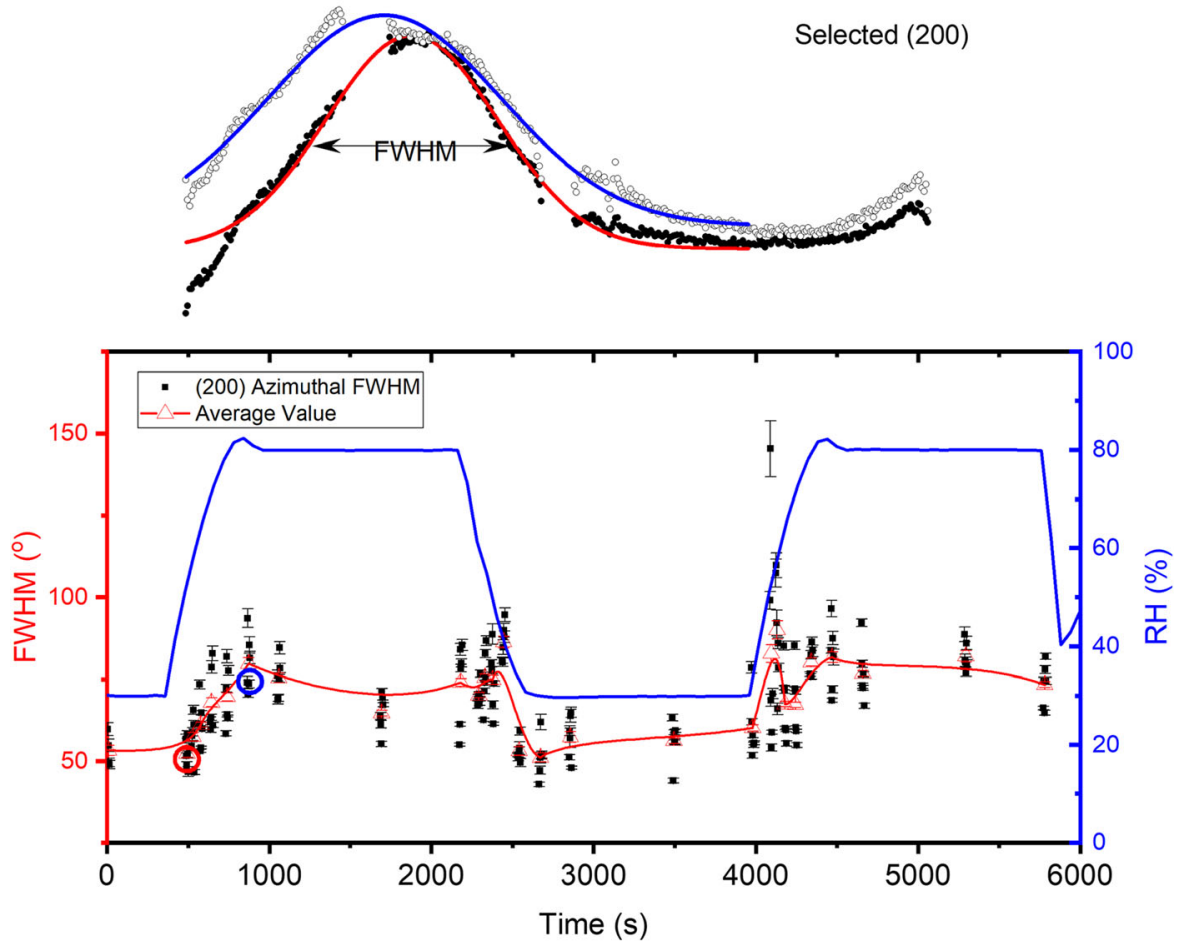

Fig. 7 Top panel: Azimuthal distribution of the (200) intensity for the narrow (black symbols/red solid line) and wide (void symbols/blue solid line) distribution examples, with the circles (red for narrow; blue for wide) marking the selected positions in the bottom panel. A Gaussian fitting curve (red) was used to

although experimental data are not available. Modelling using for instance density functional theory estimates moduli in-between the cellulose sheets to $19 \mathrm{GPa}$, in the direction of the hydrogen bonding of the extract the FWHM. Bottom panel: Summarized (200) FWHM of experimental data (black dots) and after averaging (red curve) of each set of data points at a specific time (i.e. groups of 6 black dots); blue curve shows the RH reading during the experiment (the same as in Fig. 5)

sheets to as high as $75 \mathrm{GPa}$ with a modulus in the axial direction as high as $206 \mathrm{GPa}$ (Dri et al. 2013). Anyhow, by taking only the lower values of the moduli into consideration, the forces required to deform the 
cellulose crystals to the extent observed here would still amount to about $0.22 \mathrm{GPa}$ in the axial direction and to about $0.14 \mathrm{GPa}$ in the transvers direction. Although high, these values are still below estimated values of the ultimate stress of the cellulose crystal (Mark 1967; Wu et al. 2014). The high swelling forces of wood due to moisture uptake are not a surprise, since wetting of wooden plugs have been used since ancient times to crack rocks.

The deformation behaviour observed also supports the idea that non-crystalline parts are not very frequent along the cellulose microfibrils as such areas would supposedly induce compressive strains in the longitudinal direction of the cellulose microfibrils, which is not the case observed here. The mechanism is also reflected in the azimuthal full width at half maximum (FWHM) of the (200) intensity, which is extracted from the signal intensity as a function of azimuthal angle, see Fig. 7. Given the preferred orientation of the non-crystalline cellulose and the glucomannan, the swelling will deform the lenticular amorphous polymers between the cellulose microfibril aggregates resulting in increased curvature, which shows as a broadening of the FWHM. The FWHM at the maximum swelling was 1.5 times that at the drier condition.

\section{Conclusions}

Both FTIR spectroscopy and SR-based X-ray diffraction measurements have shown that during moisture absorption the cellulose crystals are elongated and simultaneously compressed. These deformations occurred simultaneously with the increased moisture uptake and as a direct consequence of it. The deformations were also shown to be reversible. It is here hypothesised that these deformations are a consequence of the swelling forces created by the combined longitudinal and lateral expansions of the non-crystalline cellulose molecules and the glucomannan hemicellulose aligned along the cellulose crystals.

Acknowledgments This work was done within the precompetitive part of the RISE Bioeconomy Research Programme 2018-2020. The funding of the participating parties (ABInBev, BillerudKorsnäs, Georgia-Pacific, Metsä Board, MillerGraphics, MondiPowerflute and StoraEnso, is gratefully acknowledged as well as the co-financing from funds for strategic competence development to RISE from the Swedish government. The assistance of Johan Alfthan from RISE Bioeconomy and Robert Nilsson from BillerudKorsnäs in the synchrotron X-ray diffraction measurements is greatly appreciated. VINNOVA is acknowledged for financial support within the call for "Industrial pilot projects for utilisation of large-scale infrastructures for neutron and photon-based techniques". We also acknowledge DESY (Hamburg, Germany), a member of the Helmholtz Association HGF, for providing experimental facilities. Parts of this research were carried out at PETRA III and we would like to thank Dr. Matthias Schwartzkopf for assistance in using the P03 beamline.

Funding Open access funding provided by RISE Research Institutes of Sweden.. VINNOVA program "Industrial pilot projects for utilization of large-scale infrastructures for neutron and photon-based techniques".

Data availability Provided by RISE Bioeconomy and Health Box 5604, SE-11,486 Stockholm, Sweden.

Code availability Not applicable.

\section{Compliance with ethical standards}

Conflict of interest None.

Open Access This article is licensed under a Creative Commons Attribution 4.0 International License, which permits use, sharing, adaptation, distribution and reproduction in any medium or format, as long as you give appropriate credit to the original author(s) and the source, provide a link to the Creative Commons licence, and indicate if changes were made. The images or other third party material in this article are included in the article's Creative Commons licence, unless indicated otherwise in a credit line to the material. If material is not included in the article's Creative Commons licence and your intended use is not permitted by statutory regulation or exceeds the permitted use, you will need to obtain permission directly from the copyright holder. To view a copy of this licence, visit http://creativecommons.org/licenses/by/4.0/.

\section{References}

Abe K, Yamamoto H (2005) Mechanical interaction between cellulose microfibril and matrix substance in wood cell wall determined by X-ray diffraction. J Wood Sci 51:334-338

Bardage S, Donalson L, Tokoh C, Daniel G (2004) Ultrastructure of the cell wall of unbeaten Norway spruce pulp fibre surfaces. NPPRJ 19:448-452

Boyd JD (1982) An anatomical explanation for visco-elastic and mechanosorptive creep in wood, and effects of loading rate on strength. In: Baas P (ed) New perspective in wood anatomy. Martinus Nijhoff/Dr W Junk Publishing, La Hague, pp 171-222 
de Ruvo A, Lunberg R, Martin-Löf S, Söremark C (1976) Influence of temperature and humidity on the elastic and expansional properties of paper and the constituent fibre. In: Ed. F- Bolam. B.P \& B:I.F., The fundamental properties of paper related to its uses. London, UK. pp 785-806

Dri FL, Hector LG Jr, Moon RJ, Zavattieri PD (2013) Anisotropy of the elastic properties of crystalline cellulose $I_{b}$ from first principles density functional theory with Van der Waals interactions. Cellulose 20:2703-2718

Fahlén J, Salmén L (2005) Pore and matrix distribution in the fibre wall revealed by Atomic Force Microscopy and Image analysis. Biomacromolecules 6(1): 433-438

Fernandes AN, Thomas LH, Altaner CM, Callow P, Forsyth VT, Apperley DC, Kennedy CJ, Jarvis MC (2011) Nanostructure of cellulose microfibrils in spruce wood. PNAS 108(47):1195-1203

Lindh EL, Salmén L (2017) Surface accessibility of cellulose fibrils studied by hydrogen-deuterium exchange with water. Cellulose 24:21-33

Lindh EL, Terenzi C, Salmén L, Furó I (2017) Water in cellulose: evidence and identification of immobile and mobile adsorbed phases by 2H MAS NMR. PCCP 19:4360-4369

Mark RE (1967) Cell wall mechanics of tracheids. Yale University Press, New Haven

Megraw RA (1985) Wood quality factors in Loblolly pine. Tappi Press, Atlanta.

Nakai T, Yamamoto H, Nakano T, Hamatake M (2006) Mechanical behavior of the crystal lattice of natural cellulose in wood under repeated uniaxial tensile stress in the fiber direction. Wood Sci Technol 40:683-695

Nakamura K, Wada M, Kuga S, Okano T (2004) Poisson's ratio of cellulose $1 \mathrm{~b}$ and cellulose II. J Polym Sci B- Polym Phys 42:1206-1211

Nishiyama Y (2009) Structure and properties of the cellulose microfibril. J Wood Sci 55:241-249

Nishiyama Y, Langan P, Chanzy H (2002) Crystal structure and hydrogen-bonding system in cellulose $\mathrm{I} \beta$ from synchrotron $\mathrm{X}$-ray and neutron fiber diffraction. J Am Chem Soc 124:9074-9082
Olsson A-M, Salmén L (2004) The association of water to cellulose and hemicellulose in paper examined by FTIR spectroscopy. Carbohydr Res 339:813-818

Pakzad A, Simonsen J, Heiden PA, Yassar RS (2012) Size effects on the nanomechanical properties of cellulose I nanocrystals. J Mater Res 27(3):528-536

Roberts RJ, Rowe RC, York P (1994) The Poisson's ratio of microcrystalline cellulose. Int J Parmaceutics 105:177-180

Salmén L (2004) Micromechanical understanding of the cell wall structure. CR Biol 327:873-880

Salmén L (2015) Wood morphology and properties from molecular perspectives. Ann For Sci 72:679-684

Salmén L, Bergström E (2009) Cellulose structural arrangement in relation to spectral changes in tensile loading FTIR. Cellulose 16:975-982

Salmén L, Olsson A-M (2016) Physical properties of cellulosic materials related to moisture changes. Wood Sci Technol 50:81-89

Salmén L, Stevanic JS (2018) Effect of drying conditions on cellulose microfibril aggregation and "hornification". Cellulose 25:6333-6344

Stevanic JS, Salmén L (2009) Orientation of the wood polymers in spruce wood fibres. Holzforschung 63:497-503

Urquhart AR (1929) The mechanism of the adsorption of water by cotton. J Text Inst 20:T125-T132

Wickholm K, Larsson PT, Iversen T (1998) Assignment of noncrystalline forms in cellulose I by CP/MAS carbon 13 NMR spectroscopy. Carbohydr Res 312:123-129

Wu X, Moon RJ, Martini A (2014) Tensile strength of Ib crystalline cellulose predicted by molecular dynamics simulations. Cellulose 21:2233-2245

Zabler S, Paris O, Burgert I, Fratzl P (2010) Moisture changes in the plant cell wall force cellulose crystallites to deform. J Struct Biol 171:133-141

Publisher's note Springer Nature remains neutral with regard to jurisdictional claims in published maps and institutional affiliations. 